\title{
Coupled photonic crystal waveguides in hexagonal lattices
}

\author{
J.S. Brownless ${ }^{1}$, S. Mahmoodian ${ }^{1}$, K.B. Dossou ${ }^{2}$, F.J. Lawrence ${ }^{1}$, L.C. Botten ${ }^{2}$ and C.M de Sterke ${ }^{1}$ \\ 1-Centre for Ultra-high bandwidth device for Optical Systems (CUDOS), Institute for Photonics and Optical Sciences (IPOS) and \\ School of Physics, University of Sydney \\ 2-CUDOS School of Mathematical Sciences, University of Technology, Sydney
}

\begin{abstract}
We investigate the dispersion curves of coupled waveguides in hexagonal lattices. We show that hexagonal PCW lattices have coupling coefficients that change magnitude and sign along the BZ. It is also shown that the modes of these structures no longer form odd and even modes but are superposed in a more general sense.

OCIS codes: (130.5296) Photonic crystal waveguides; (130.2790) Guided waves; (260.2110) Electromagnetic optics; (350.4238) Nanophotonics and photonic crystals; (050.5298) Photonic crystals; (230.5298) Photonic crystals; (160.5298) Photonic crystals.
\end{abstract}

Coupling of waveguide modes is a common phenomenon in photonics and has been investigated in the linear [1] nonlinear [2] regimes. Though the properties of every waveguide type can be tailored, Photonic Crystal Waveguides (PCWs) are particularly versatile since the many degrees of freedom allow for very delicate adjustment of their dispersion relation. Coupled PCWs have been used to create a variety of devices including efficient slow light couplers [3], coupled PCWs with a large bandwidth-delay product [4], as well as PCWs with negative discrete refraction [5]. It has been shown [6] that coupled PCWs in square PC lattices, unlike conventional waveguide, have a fundamental mode that is not always an even superposition of the single PCW modes but is either odd or even depending on the number of rows separating the PCWs. This is important in determining the coupling coefficient of PCWs as this is related to the propagation constant of the modes via $C=\left(\beta_{\text {even }}-\beta_{\text {odd }}\right) / 2$ [7].

Since PCWs are typically fabricated in hexagonal lattices, we here consider the properties of PCWs in such lattices. To our knowledge, this issue has not been addressed in general. Here we present the physics of the modes of coupled waveguides in hexagonal PCs and show that it is much richer than in square lattices. As illustrated in the insets of Fig 1, there are two distinct geometries in the hexagonal case, when the two waveguides are aligned ("in-line"), and when they are out of line ("staggered"). It is apparent that these have different symmetry properties, with the in-line geometry having an up-down symmetry which is absent in the staggered geometry. Dispersion curves arising from the two geometries are shown in Figure 1. Each PCW is defined by altering the refractive index in a row of air holes; an index of $n=1.5$ (in a background of $n=3.0$ ) is chosen to ensure that the coupling of the waveguides is sufficiently large to be easily visible on the dispersion curves even for large separations between the waveguides. We note that this particular choice does not affect the qualitative aspects of the results discussed below.

Conventionally, the dispersion relations of two coupled waveguides tend to run approximately parallel to each other, with, at fixed wavenumber $k$, their spacing determined by degree to which the modal fields are coupled. Figs 1 show that for $k_{x} d>2$ the dispersion relations exhibit serpentine behavior, crossing each other several times, in contrast to the dispersion curves in uniform media and in square lattices. This means that not only does the magnitude of the coupling coefficient change along the Brillouin zone (BZ), its sign changes and it vanishes at the crossings. A key difference between the in-line and staggered geometries is the degeneracy in the staggered case at the BZ edge. This non-accidental degeneracy arises as a result of the glide-plane symmetry in the staggered case. Note that, in contrast, for $k_{x} d<2$ no crossing occur.

We can obtain some insight into this behaviour of the dispersion curves for the coupled hexagonal PCWs using a tight-binding treatment [1]. In this treatment, the coupling of the modes is proportional to the overlap integral

$$
\int_{W G 1} \mathbf{E}_{1}^{*}(\mathbf{r}) \cdot \mathbf{E}_{2}(\mathbf{r}) d \mathbf{r}
$$

Thus coupling depends on the product of the field of the two waveguides, integrated over one of holes of one of the waveguides - in effect this means that the coupling is determined by the way the field in the direction orthogonal to a waveguide. For square PCWs, depending on the geometry, the PCW mode either does or does not change sign sign after each period in the direction transverse to the waveguide [6]. Thus, the sign of the overlap integral (2) changes depending on the separation of the PCWs, determining whether 


\section{FThJ3.pdf}
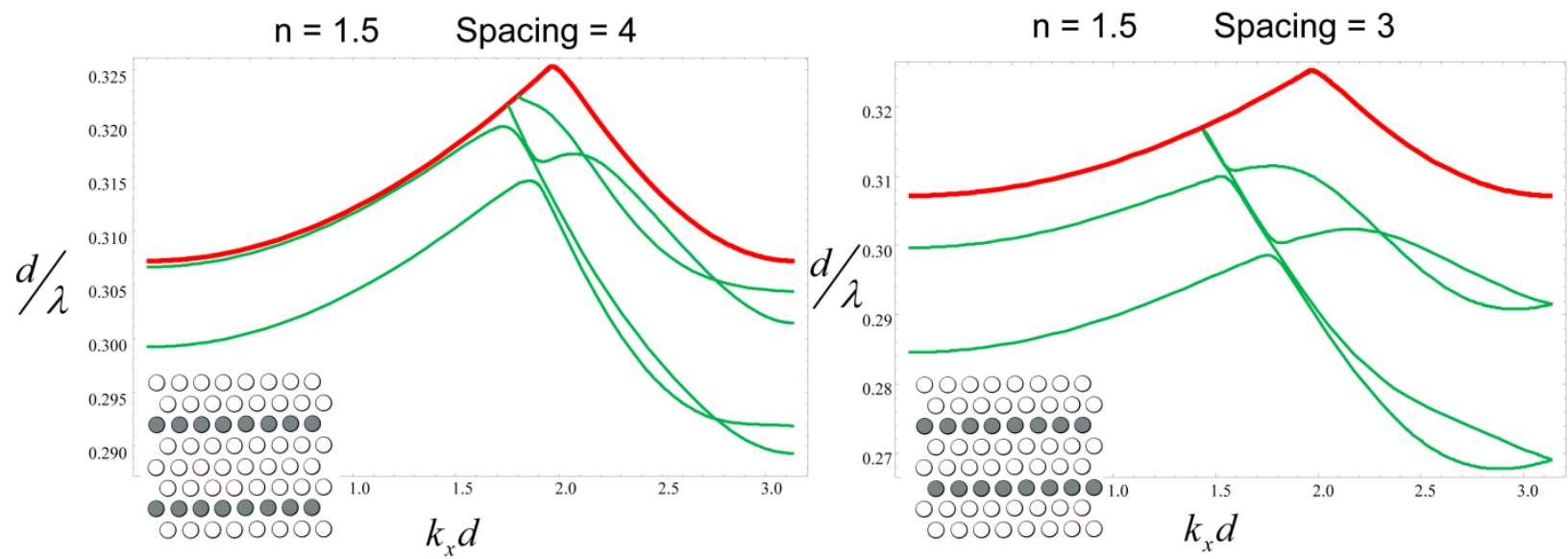

Fig. 1. Dispersion curves of coupled PCWs in hexagonal lattices for an in-line (left) geometry and a staggered (right) geometry as shown in the insets. Green lines indicate dispersion relation for coupled PCW modes, while the red line indicates the projected band-edge. Here the modes are TE polarized ( $\mathrm{H}$ out of plane) with a background index of the photonic crystal, $\mathrm{n}_{\mathrm{b}}=3$ and the holes have an index of $\mathrm{n}_{\mathrm{h}}=1$ and a radius of $\mathrm{a}=0.3 \mathrm{~d}$, where $\mathrm{d}$ is the period. The waveguides are constructed by changing the refractive index of the holes to $\mathrm{n}_{\mathrm{w}}=1.5$, which we chose to ensure the fields have sufficient overlap

the fundamental mode is even or odd. For $k_{x} d<2$ the behavior in a hexagonal lattice is similar to that in a square lattice and, as confirmed in Figs 1 the crossing do not occur [8]. At the BZ edge the field changes sign after two periods and so the sign of the field is unchanged after four periods. For intermediate values of $k_{x} d$ the field evolves gradually between these two cases and the field acquires an arbitrary phase after traversing a single period. This leads to a winding up of the phase between $k_{x} d \sim 2$ and $k_{x} d=\pi$, leading to the crossings observed in Figs 1 . This is consistent with the observation in Fig 2 that with increasing distance between the waveguides the additional crossings originate from the BZ edge, and then shift to towards the $\mathrm{BZ}$ centre.

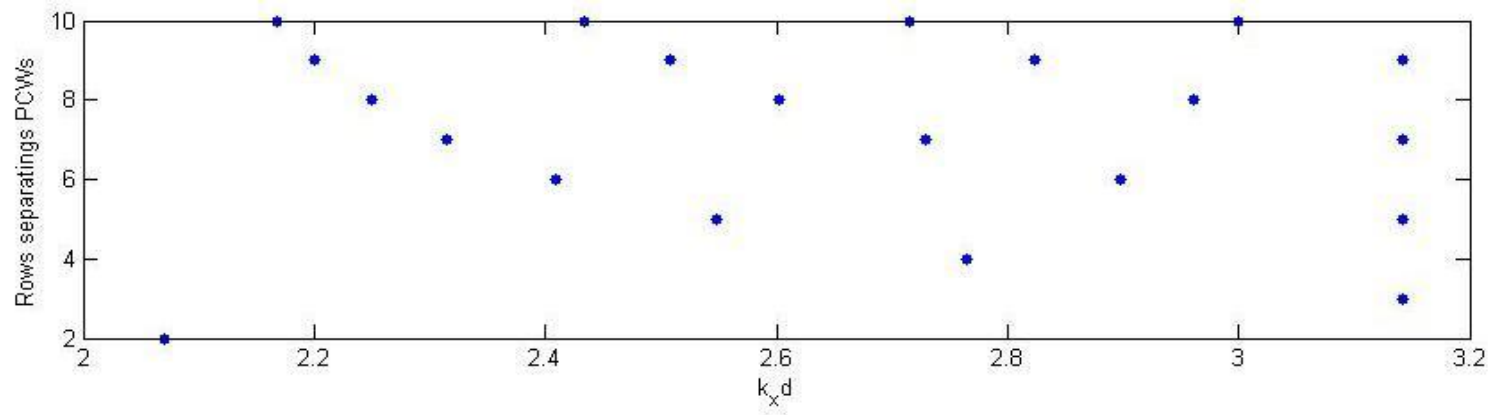

Fig. 2. Positions of the crossings of the dispersion relation in the $B Z$ for the even mode (lowest two curves in Fig. 1) waveguide withseparations varying from $2-10$.

Thus, we have examined the dispersion relations of PCW waveguides in hexagonal lattices. These have unique features which do not occur in conventional waveguides or in square lattice PCWs. The serpentine behavior of the dispersion curves is associated with a coupling constant which changes not only in magnitude but also in phase along the BZ. Though we cannot discuss this here in detail, the associated fields are neither odd or even superpositions of the individual PCW modes but are more generally be expressed as

$$
|\psi\rangle=\left|\psi_{1}\right\rangle \pm e^{i \phi}\left|\psi_{2}\right\rangle
$$

where $\left|\psi_{\mathrm{i}}\right\rangle$ indicates the field in the uncoupled PCWs.. Here, $\varphi=k_{x} l d / 2$, where $l$ is the number of rows separating the PCWs and $d$ is the period of the PCW.

\section{References}

[1] Pochi Yeh, Optical waves in layered media, (Wiley New York, 1988)

[2] H.S. Eisenberg et al, "Discrete spatial solitons in waveguide arrays," Phys. Rev. Lett. 81, 16 3383-3386 (1998)

[3] S. Ha et al, "Dispersionless tunneling of slow light in antisymmetric photonic crystal couplers," Opt. Exp. 16 1104-1114 (2008)

[4] T. Baba, "Slow light in photonic crystals," Nature Photonics 2, 465-473 (2008)

[5] A. Locatelli et al "Discrete negative refraction in photonic crystal waveguide arrays," Opt. Lett. 319 1343-1345 (2006)

[6] C.M. de Sterke et al, "Modes of coupled photonic crystal waveguides," Opt. Lett. 29, 12 1384-1387 (2003)

[7] A. Locatelli et al "Diffraction engineering in arrays of photonic crystal waveguides," Opt. Lett. 3021 2894-2896 (2005)

[8] S. Mahmoodian et al "Modes of shallow photonic crystal waveguides: Semi-Analytic Treatment," 1722 19629-19643 (2009) 